\title{
Erratum: Classical integrability for three-point functions: cognate structure at weak and strong couplings
}

\author{
Yoichi Kazama, ${ }^{a, b, c}$ Shota Komatsu ${ }^{d}$ and Takuya Nishimura ${ }^{c}$ \\ ${ }^{a}$ Research Center for Mathematical Physics, Rikkyo University, \\ Toshima-ku, Tokyo 171-8501, Japan \\ ${ }^{b}$ Quantum Hadron Physics Laboratory, RIKEN Nishina Center, \\ Wako 351-0198, Japan \\ ${ }^{c}$ Institute of Physics, University of Tokyo, \\ Komaba, Meguro-ku, Tokyo 153-8902, Japan \\ ${ }^{d}$ Perimeter Institute for Theoretical Physics, \\ 31 Caroline Street North, Waterloo, Ontario, N2L 2Y5, Canada \\ E-mail: yoichi.kazama@gmail.com, skomatsu@perimeterinstitute.ca, \\ tnishimura@hep1.c.u-tokyo.ac.jp
}

ERRATUM TO: JHEP10(2016)042

KEYwORDS: AdS-CFT Correspondence, Integrable Field Theories

ARXIV EPRINT: 1603.03164

1. In the equation (7.34) on page 49 , the term that contains $\operatorname{Li}_{2}\left(e^{i \hat{p}_{1}+i \hat{p}_{2}+i \hat{p}_{3}}\right)$ was missing on the first line. The correct formulae, which follow from the preceding discussions in the paper, are

$$
\begin{aligned}
(\mathcal{L}+\mathcal{R})_{\mathrm{AdS}} & =\oint_{U} \frac{d u}{2 \pi} \operatorname{Li}_{2}\left(e^{i \hat{p}_{1}+i \hat{p}_{2}+i \hat{p}_{3}}\right)+\sum_{\{i, j, k\} \in \operatorname{cperm}\{1,2,3\}} \oint_{U} \frac{d u}{2 \pi} \operatorname{Li}_{2}\left(e^{i \hat{p}_{i}+i \hat{p}_{j}-i \hat{p}_{k}}\right), \\
\mathcal{N}_{\mathrm{AdS}} & =-\sum_{k} \oint_{U} \frac{d u}{2 \pi} \operatorname{Li}_{2}\left(e^{2 i \hat{p}_{k}}\right) .
\end{aligned}
$$

2. We inadvertently wrote an incorrect formula for the equation (7.37) on page 50. The correct formula which is consistent with other formulae in the paper is

$$
p_{i}(x) \sim-\frac{i \Delta_{i}}{4 g \theta}+O(\theta) .
$$


3. In the equation (7.49) on page 52 , the term that contains $\operatorname{Li}_{2}\left(e^{i p_{1}+i p_{2}+i p_{3}}\right)$ was missing on the first line. The correct formulae, which follow from the discussions in the paper, are

$$
\begin{aligned}
(\mathcal{L}+\mathcal{R})_{\mathrm{S}} & =\oint_{U} \frac{d u}{2 \pi} \operatorname{Li}_{2}\left(e^{i p_{1}+i p_{2}+i p_{3}}\right)+\frac{1}{2} \sum_{\{i, j, k\} \in \operatorname{cperm}\{1,2,3\}}\left(\oint_{\Gamma_{i} \cup \Gamma_{j} \cup 2 U} \frac{d u}{2 \pi} \operatorname{Li}_{2}\left(e^{i p_{i}+i p_{j}-i p_{k}}\right)\right) \\
\mathcal{N}_{\mathrm{S}} & =-\frac{1}{2} \sum_{k} \oint_{\Gamma_{k} \cup 2 U} \frac{d u}{2 \pi} \operatorname{Li}_{2}\left(e^{2 i p_{k}}\right) .
\end{aligned}
$$

Open Access. This article is distributed under the terms of the Creative Commons Attribution License (CC-BY 4.0), which permits any use, distribution and reproduction in any medium, provided the original author(s) and source are credited. 